\title{
Smarter, faster, better vaccines
}

\author{
Evin Allen
}

School of Pharmacy, UCC

\section{Vaccines}

Since Edward Jenner pioneered modern vaccination in the late 18th century, no other public health intervention has saved as many lives. Vaccines keep us safe and dramatically reduce the mortality and morbidity from many infectious diseases such as measles, diphtheria and influenza. Vaccines are safe, non-viable versions of pathogens (viruses, bacteria) that when administered directs the body into producing an immune response against the pathogen, and it is this that prevents any subsequent infection by the live bacteria or virus.

\section{Vaccine Administration}

Vaccines are composed of proteins, unlike most medicines that we are prescribed which are chemical molecules produced synthetically by a series of chemical reactions. Chemical molecules are quite small in size and are very stable to the environment; in contrast proteins are large and complex and very unstable. This means that generally we can't give vaccines as tablets because they would be degraded in the stomach by the same enzymes that break down our food. In addition, we can't administer vaccines as creams or patches because they are too big to pass through the skin unlike small drugs such as nicotine (used for smoking cessation) or voltarol ${ }^{\circledR}$ (used for pain treatment). This results in vaccines being administered as a liquid solution using the often dreaded needle and syringe.

Ultimately, this necessitates a trained healthcare professional i.e. doctor, nurse or pharmacist to administer the vaccine. This adds significant cost to the immunisation process, limits the availability and uptake of vaccines while decreasing the patient experience. In low resource settings, access to well-trained healthcare staff is often low and this can result in erroneous vaccine administration leading to suboptimal patient outcomes. The use of needles and syringes also generates biological waste (contained in the yellow and orange bins in the doctors' surgery). This is expensive to dispose of and can be dangerous if not disposed of due to the risk of cross contamination from potential reuse or accidental stabbing. Thus it is safe to say giving vaccines using a needle and syringe is a last resort for both patients and practitioners! 


\section{Vaccine Distribution}

The poor stability of vaccines when exposed to extremes of temperature and humidity constrains how we ship and store vaccines. Unlike most tablets and creams, liquid vaccines need to be refrigerated at all points between manufacture and administration. This obligates the use of costly, complex refrigerated distribution and storage systems. It is estimated that this accounts for up to $80 \%$ of the cost of immunisation programmes. In remote, low-income areas the infrastructure to maintain this constant refrigerated environment or "cold-chain" is sadly lacking or not fit for purpose. This results in $25-50 \%$ of vaccines manufactured annually being wasted due to a break down in the cold chain. This has prompted significant research into developing heat-stable vaccines and is part of the UN Millennium Development Goals and the Bill and Melinda Gates Foundation programme aims.

\section{Microneedle Vaccine Patches}

Any solution to the issue of ineffective vaccine delivery should circumvent the distribution and administration barriers outlined; basically vaccines should be easy to use and should be distributable at ambient temperatures. An innovative solution, under development in the UCC School of Pharmacy in collaboration with the Tyndall National Institute, is the use of microscopic projections or microneedles on a flat patch to deliver vaccines through the skin. The difference between our vaccine patch and conventional flat patches are the ultra-sharp yet ultra-small microneedles on the patch surface. Upon application to the skin, these microneedles pierce the tough outer layer of the skin allowing the delivery of the large vaccine molecules deep within the skin. Innovatively, the vaccine itself is contained within the needle structure and dissolves into the skin once the patch is applied. In contrast to a flat patch and similar to a needle, this technology does not rely on skin permeation rather skin penetration ensuring that vaccines are delivered to the body.

a)

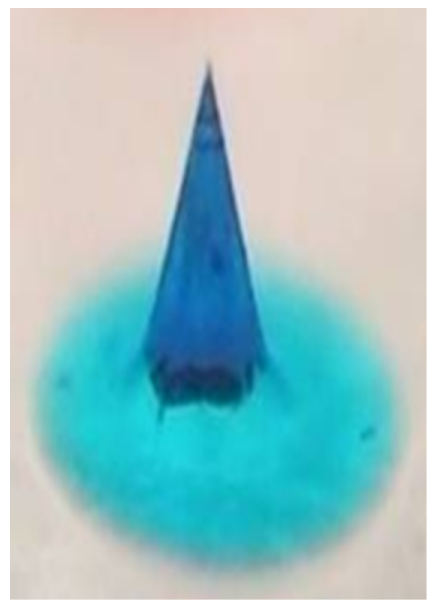

b)

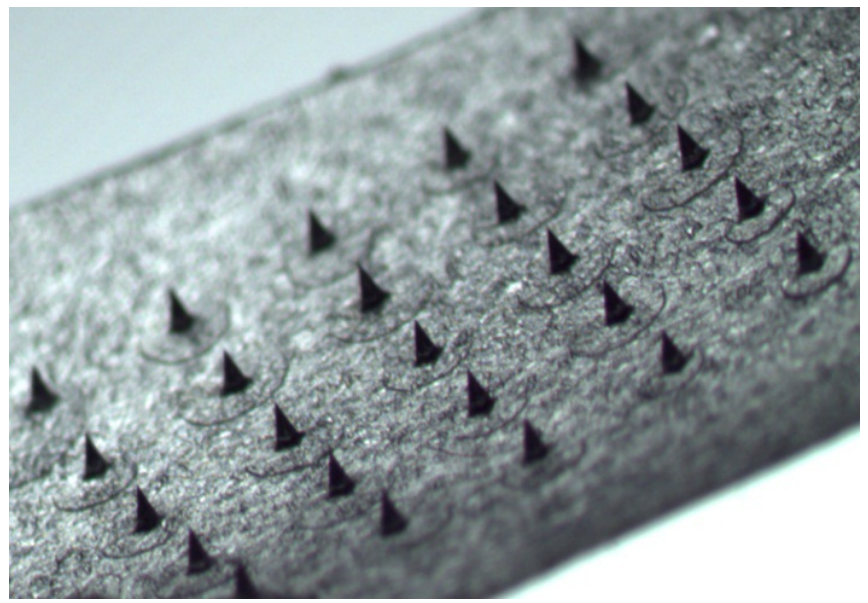

Figure 1: A, 1B: a) An indiviudal microneedle $0.5 \mathrm{~cm}$ in height. b) Typical microneedle vaccine patch $1 \mathrm{~cm}^{2}$ in size. Source: Author. 
Source: AuthorMicroneedles are usually half a centimetre or less in height (figure 1) and do not penetrate deep enough within the skin to activate our pain receptors or damage blood vessels within the skin. This minimally invasive method of vaccine delivery avoids the pain often associated with traditional vaccination. Microneedle mediated vaccine delivery also removes patients fears of needles that may be preventing them getting vaccinated. Due to their simple design, microneedles patches can be self-administered by patients and overcomes the need for trained professionals. Microneedles are a solid dosage form and therefore should demonstrate enhanced heat stability compared to liquid solutions. This assumption is based on the fact that protein solutions such as milk rapidly go off if not refrigerated but can be stored for much longer periods of time if dried to a solid form such a milk powder.

\section{Influenza Vaccine Microneedle Patch}

My research focuses on the development of an influenza vaccine microneedle patch to allow for immunisation against seasonal 'flu. The hypothesis was that we could successfully reformulate a licensed commercial seasonal flu vaccine into a microneedle vaccine patch that would be amenable to self-administration and ambient shipping conditions.

\section{Vaccine reformulation}

So it's easy to take a liquid vaccine and make it into a microneedle patch, right? Not really, making medicines that are safe, stable and efficacious is an extremely challenging area known as formulation science. Some substances dislike water; others dislike acidic or basic solutions and require a certain a $\mathrm{pH}$ to work whereas some can interact with inert materials within the "formulation" causing them to lose their function. Every medicine that we take is a complex recipe that contain various different ingredients, some are added for flavour (for example, in children's medicines), some to preserve the medicine, some to stabilise the drug or vaccine that we are delivering. In addition, all the materials that we use in our formulation need to be certified as safe. Thus as formulation scientists we are like chefs with a very small number of ingredients expected to make the same high quality dish without losing any of the taste. The first part of my project was to develop a "secret sauce" that would be compatible with and stabilise the vaccine but would form needles that would be strong enough to pierce the skin.

This series of limitations lead me to develop a formulation based on the sugar trehalose. Trehalose is a naturally occurring safe, sugar substance, very similar to glucose (the main ingredient in the drink Lucozade), which is used by many plants and insects to survive. When these plants or animals are dehydrated, trehalose takes the place of the water molecules that have been lost and secure the physical integrity of the animal or plant. Upon rehydration, trehalose is replaced by water allowing for normal biological function 
to resume. Similarly, when we dry vaccine solutions, trehalose takes the place of water ensuring that the vaccine proteins aren't destroyed.

As vaccines are quite sensitive to heat and other physical stresses, we have to use low temperatures and gentle processes to manufacture microneedle patches. To make a bun you add your mixture to the baking tray where it takes the shape of the tray forming a lovely bun. In our kitchen we use a specialised baking tray or mold, designed and fabricated by engineers in Tyndall using a state of the art, high precision patented process. These molds produce ultra-sharp microneedles which are able to penetrate skin easier than other microneedle designs because the application pressure is focussed on a very small area (like a nail or a stiletto). I borrow a technique from cooking whereby a modified pressure cooker is used to dry the vaccine after is dispensed to the mold, so that we can dry the vaccine at room temperature rather than at high temperature. Having tested the integrity of the vaccine pre and post fabrication I found that this process had no effect on the vaccine. This is a vital step in proving to regulators that microneedles are a safe and effective alternative to liquid vaccines.

\section{Do Microneedles work?}

Do microneedles work? This is a fundamental question that has kept me up all night thinking about science over the last three years. Do microneedles penetrate the tough skin that protects us from external insults? Is skin the right place to delivery vaccines to, considering we normally inject vaccine into the muscle? To answer these questions, I've performed an extensive number of tests in mice and pigs as well as lab based tests. We use animal tests to provide information that in vitro tests will never supply, mice are chosen as they are easy to handle and have a long track record of use in early stage vaccine research.

Initially I tested the effectiveness of microneedle vaccines in mice by administering equal amounts of vaccines using our vaccine patch and by the traditional needle and syringe. The results were staggering, it was observed that a much higher response was observed in mice immunised using the vaccine patch. Subsequently, mice were immunised intramuscularly with needle and syringe with higher doses of vaccine and it was found that eight times the dose administered via our patch system was required to get equal responses. I also examined different formulations to determine the best secret sauce, by adding various plastic polymers and sugars such as polyvinyl alcohol, sucrose (i.e. table sugar) etc. to the formulation and found that polymers while making microneedles mechanically stronger delay how quickly vaccines are dissolved. From a patient perspective this is really undesirable as we want vaccines to be delivered quickly as this will mean patients wearing patches for shorter times. This minimises the risk of poor outcomes from patches falling off or being removed too quickly, as nobody likes wearing patches or plasters for a period of time.

These results demonstrate the enormous potential that microneedle vaccines have as well 
as the attractiveness of skin for vaccine delivery. Skin is the largest organ of the body and has a rich immune system of its own. It is this high quality immune system that drives the enhanced vaccine response we see compared to intramuscular needle based delivery. In practice, we only rarely observe the use of vaccine delivery to the skin (intradermally) for the BCG (Tuberculosis) vaccine, due to the extremely challenging aspect of delivering vaccines intradermally.

\section{Microneedle vaccines: Universal vaccines}

Every year, the circulating flu viruses changes slightly (a phenomenon known as drift) and in rare cases they dramatically change (shift), this dramatic change often results in an influenza pandemic like the swine flu outbreak. This constant viral evolution necessitates the development of a new vaccine every year. Wouldn't it be great if we had a universal flu vaccine that would protect against all strains of flu? Scientists have been intensely working on this Holy Grail for years with little success. In collaboration with a virology group based in the UK, we studied the responses induced by microneedle vaccination against a diverse group of flu strains. Intriguingly, we found that the responses in mice using vaccine patches neutralised vastly different influenza viruses unrelated to the strain within the vaccine. This is a very novel and promising finding and suggests that delivering vaccines via an alternative route such as the skin results in a much broader, better response.

\section{Vaccine Stability}

The major driver for a change in how we deliver vaccines is the need for thermostabilisation. I studied the stability of vaccines at accelerated storage conditions i.e. $40^{\circ} \mathrm{C}$ and $75 \%$ relative humidity. Vaccines formulated in our microneedle patch system were found to be very stable and did not lose any activity over a 12 month period; in contrast liquid vaccine began to degrade after 10 days. As microneedles rely on their unique design to be effective in vivo, we also examined the immune response when microneedles on stability study were used to immunise mice. Excitingly, no decrease in immune response was observed compared to patches that had not been exposed to these extremes of conditions.

\section{What does all this mean?}

As a PhD student, one becomes very philosophical over time and begins to ask profound questions such as what does all this mean? Does it mean anything? Have I added anything to existing knowledge? Thankfully these results do mean something in this field. The fact that we get equivalent responses with lower vaccine doses when using a microneedle patch means that we can immunise greater numbers of people with the same amount of starting material. By generating a broad immune response, we have a vaccine that could protect against viruses different to that contained in the vaccine itself. We also have developed a vaccine delivery system that harnesses the rich and underexploited immune system within 
our skin. Most importantly we have a vaccine patch that is highly stable to extremes of temperature and humidity allowing for ambient shipping. It means that one day you will be able to get vaccines delivered in the post that you can self-administer without the need for a healthcare professional.

\section{The future of Microneedles}

Microneedle mediated drug and vaccine delivery has become an area of rapid interest with many pharmaceutical and biotech companies collaborating with university research groups or developing their own research units. The commercial success of microneedles vaccines is dependent on significant investment into clinical trials and manufacturing facilities. Clinical trials will initially aim to demonstrate the safety of vaccine patches in small numbers of patients. This will be followed by larger trials that will recruit thousands of patients to show how well microneedles will work compared to traditional liquid vaccines. Existing vaccines are relatively low cost pharmaceutical products compared to some of the new oncology or hepatitis medicines being marketed. This means that vaccine patches will need to be manufactured at equal or lower prices for companies to change the way they make vaccines. It is healthcare systems, patients and providers such as the HSE or WHO that incurs the cost for vaccine distribution and administration, so manufacturers need to be incentivised to bring this technology to market. To do this, innovative technologies will need to be harnessed to ensure that we can bring this disruptive vaccine delivery product to market. This issue has been significantly researched as part of my research, with the development of a system that uses existing inkjet printing technology.

This project demonstrates the tangible effect that university based research can have on healthcare and patient outcomes. Any death is sad, but vaccine preventable deaths are beyond sad; they are inexcusable and unforgivable. Hopefully in the near future, these smarter, faster, better microneedle vaccines will end this tradgedy.

Thanks to my supervisor Dr Abina Crean and co-supervisor Dr Anne Moore and Tyndall collaborator Dr Conor O'Mahony. In particular, I would like to acknowledge the microneedles research group in the School of Pharmacy and the technical staff for their on-going support. I would also like to acknowledge the School of Pharmacy and COMH for my PhD funding. 